\title{
Improving access to child health care in Indonesia through community case management
}

\begin{abstract}
Objectives - In order to reduce infant mortality in Indonesia, Community Case Management (CCM) was introduced. CCM is a community-based service delivery model to improve children's wellness and longevity, involving the delivery of lifesaving, curative interventions to address common childhood illnesses, particularly where there are limited facility-based services. This paper reports the findings of a qualitative study that investigated the implementation of CCM in the Kutai Timur district, East Kalimantan Indonesia from the perspective of mothers who received care.
\end{abstract}

Methods - Seven mothers and health workers were observed during a consultation and these mothers were interviewed in their home weeks after delivery. Field notes and the interview transcriptions were analysed thematically.

Findings - Mothers reported that their access to care had improved, along with an increase in their knowledge of infant danger signs and when to seek care. Family compliance with care plans was also found to have improved. Mothers expressed satisfaction with the care provided under the CCM model. The mothers expressed a need for a nurse or midwife to be posted in each village, preferably someone from that village. However two mothers did not wish their children to receive health interventions as they did not believe these to be culturally appropriate.

Conclusions - CCM is seen by rural Indonesian mothers to be a helpful model of care in terms of increasing access to health care and the uptake of lifesaving interventions for sick children. However there is a need to modify the program to demonstrate cultural sensitivity and meet cultural needs of the target population. While CCM is a potentially effective model of care, further integrative strategies are required to embed this model into maternal and child health service delivery.

Key words - child health, access, mothers, community case management

Significance - Understanding CCM from the perspective of families is important to ensure service is appropriate, acceptable and accessible to users to guarantee uptake, equitable service delivery and efficiency. These insights are important to inform policy decisions and sustain improvements in practice and ultimately child health outcomes.

\section{Introduction}

The high infant mortality rate (IMR) in low and middle income countries (LMIC) remains a global health issue. Every year almost seven million children die before reaching their fifth birthday, $73 \%$ of these deaths occurred within the first year of life [1]. Indonesia has been demonstrating progress in reducing the IMR in the last decade. In 2015, the country had successfully reached a figure of 23 infant deaths per 1,000 live births from a staggeringly high 37 per 1,000 in 2005 [1, 2]. Most childhood mortality in the country occurs during infancy due to neonatal problems such as respiratory failures, low birth weight, premature births, and neonatal infections [3]. In order to support the global effort to reduce infant mortality, the World Health Organization (WHO) and the United Nations Children's Fund (UNICEF) developed a policy highlighting the importance of communitybased treatment and the enhancement of quality service at primary care level. One initiative associated with this policy is community case management (CCM). 
CCM is a community-based service delivery model designed to improve children's wellness and longevity [4]. The model involves the delivery of lifesaving, curative interventions to address common childhood illnesses such as diarrhoea, pneumonia and malaria, particularly where there are limited facility-based services. [5]. CCM involves the delivery of evidence-based child survival interventions [6] that include, antibiotics, oral rehydration therapy, zinc, and antimalarial drugs. CCM also includes timely care seeking, promoting appropriate home care, and referral to facilities [5]. CCM has been implemented in a number of countries [7-9]. In CCM, primary health care workers (PHCWs) (nurses, midwives and trained health volunteers), provide health services at the community level through family visits or consultations at village clinics to bring the service closer to the community and provide timely and accessible care. While nurses and midwives had a minimum of three years nursing and midwifery training, health volunteers graduated from high schools only. All PHCWs undertook a two weeks training program prior the implementation of CCM.

While there is strong medical evidence regarding the need for and success of the package of care delivered as part of the CCM model in reducing infant mortality [10-12], and the benefits received by the community [9], there is little knowledge concerning the experience of families who receive this care. Understanding CCM from the perspective of families is important to ensure service is appropriate, acceptable and accessible to users to guarantee uptake, equitable service delivery and efficiency. In addition, an investigation into community experiences of models of care is an important component of assessing the merit of health services and documenting lessons learnt to inform policy decisions and sustain improvements in practice and ultimately child health outcomes [9].

This paper reports research that investigated the experiences of families who formed part of the implementation of the CCM model of care. The model was aimed at treating childhood illnesses in the rural area of the Kutai Timur district, East Kalimantan. This paper provides a rich description of how the CCM program was received in the Kutai Timur district and how mothers perceive its benefits.

\section{Kutai Timur: the context}

Kutai Timur is located in the province of East Kalimantan, Kalimantan Island; the biggest island in Indonesia. With a size of $35,747 \mathrm{~km}^{2}$, the district is inhabited by 253,847 people [13], or approximately seven people living in every $\mathrm{km}^{2}$. The population has grown by about four percent every year as the result of the increase in the number of births, and the number of people who come to work and migrate due to the government transmigration program. Kutai Timur consists of 18 subdistricts with 135 villages. Health care delivery in Kutai Timur is managed by a district health office, with one public hospital and 19 Puskesmas (community health centres) [14]. However not all were in operation at the time of the study due to a shortage of resources and staff [15]. Health services are mainly provided by medical doctors, nurses and midwives [14]. The distribution of health workers across the district is challenging. CCM was implemented in 17 villages in seven subdistricts. At the time of this study five of seventeen villages had neither a doctor, a nurse nor a midwife in residence [14]. Traditional healers and traditional birth attendants known as Dukun [7] or lay health workers also provide health care at the village level.

Accessing a health facility in Kutai Timur can be a challenge for many communities as villages may be geographically isolated. Many of the roads between villages are not sealed and travel can be further complicated by the absence of public transport and the cost incurred by this travel. Some villages are separated from the main island by a sea strait and others by rivers. To access Puskesmas, people often needed to hire a boat, which is expensive.

\section{Methods}

In order to comprehensively consider the impact of CCM on families from the mother's perspective, qualitative data were collected from July to October 2011. Ethical clearance was obtained from the UTS Human Ethics Research Committee as well as from the Faculty of Nursing Universitas Indonesia. The data collection included an observation of seven consultations between PHCWs and mothers followed by in depth interviews with these mothers after the consultation to understand their experiences of receiving care. Mothers were purposively 
selected [16] as they are the primary carers of their sick children and therefore have most contact with PHCWs delivering CCM. One mother was selected from each of the seven villages where CCM was implemented.

PHCWs asked mothers who approached them for a non-urgent health consultation if they would like to take part in the study. Once a mother showed interest the first author provided information and obtained consent. Seven mothers were recruited in the study. Mothers were aged between 18 and 37 with infants aged 1 to 4 weeks. Five mothers had completed primary school while two had not. All mothers were housewives.

Observations were conducted during the PHCWs' consultation. The purpose of the observation was to capture the interaction between the PHCW and mother, the intervention delivered by PHCWs using CCM protocols and how mothers responded to the care they received. In depth interviews of approximately one hour were held with mothers after the consultation. Further observation and interviews were terminated when data saturation was achieved and no new understandings were emerging. The interviews were recorded and the observations were written up as field notes. While field notes are written in English, the interviews were conducted and transcribed in Bahasa Indonesia. The transcripts were then translated into English with the closest interpretation and meaning for further analysis. As a native Indonesian, the author used the approach of 'researcher as translator' [17]. In this role, the author was able to pay close attention to cross cultural meanings and interpretations, and therefore engaged with the issues of meaning equivalence within the research process [17]

The data were analysed using thematic analysis [18]. Once collected, the data were reviewed, sorted and classified to identify issues, topics, patterns, and themes. This method of analysis provided an opportunity for the researcher to become immersed within the data and so generate rich insights into the participants' world [18]. In this study transcripts were read repeatedly (reread) and sorted to allow the creation of a conceptual map of predominant story lines. As new data was obtained through the continued process of interview and observation, new categories were created and some categories collapsed into themes. Categories developed into themes by virtue of their fit with and truthfulness to the data $[19,20]$. Field notes were used alongside interview data to link the context with phenomena and improve rigor of the research.

\section{Findings}

Four themes emerged from the data. These themes were geography and services; child health knowledge; satisfaction to care and services, and a theme that focuses on socio cultural context issues. These are described below.

\section{Geography and services}

Participants noted that services were closer to home. One participant said that, "with the presence of a nurse and midwife (PHCWs) in our village, the service becomes closer and it is easier for us to get health services" [Mother, with son age 4 weeks]. Mothers said that since the delivery of CCM they did not need to take their sick children to town for care. A mother participant said:

I am satisfied ... with the nurse and midwife here, it is easier for us to get service ... we used to go to (local town) when we need a medical service for our sick babies ... it now becomes easier ... (to get service) was not difficult ... very convenient and close. [Mother, with son age 3 weeks]

A mother also confirmed that before a PHCW was employed in her village, she used to seek help from a traditional birth attendant as there was no one else available. Since the introduction of CCM in her village, the mother reported that she would rather come to the PHCW for help. Care is even more available to mothers because with CCM, PHCWs actively visited families with sick babies to their homes. The presence of PHCWs in their villages was said by mothers to have reduced delays in receiving medical care. One mother reflected upon the experience of her neighbour whose baby died on the way to the health centre due to the distance and lack of transport.

\section{Child health knowledge}

Mothers reported that their understanding of child health had improved following the CCM implementation. Mothers stated that home visits from PHCWs had helped them to learn about infant dangers signs and know when to seek care. Some mothers reported sharing this new knowledge with others in the community and said 
they felt confident to either perform child health practices or ask a health worker for help with a sick child. This was verified by one mother who said:

This is new experience that I can share with my relatives ... in case I have a new baby with low birth weight again (in the future); I know what to do ... This is really good because I have never received service like this before. [Mother, with son age 4 weeks]

The interview data was corroborated with data from non-participant observation of mothers with the PHCW. One mother with a low birth weight baby demonstrated the essential newborn care practice of Kangaroo Mother Care that was taught to mothers as part of the CCM protocol. Another mother recalled appropriate umbilical cord care that was also part of the CCM community education efforts.

The periodic home visits that PHCWs made to mothers was said to have improved the mothers' compliance with prescribed treatments. During a follow-up visit by a PHCW (midwife) to a mother with a baby that had an eye infection it was observed that the mother had dispensed the antibiotic eye drops as evidenced by the improved condition of the baby's eyes and reduction in the amount of the medication. Three mothers also reported that they had followed the protocols taught by the PHCWs. They said that from that time on they always washed their hands before touching their babies. In the interview following the visit, one PHCW affirmed that "....and we can monitor the mothers' compliance with treatment because the mother is the one who gives the medication to the sick baby at home...we can ask to the mother (how the medication was administered). Before CCM (was implemented), we had never known how the follow up care at home was (by mother)...we couldn't monitor. “

\section{Satisfaction to care and services}

Mothers indicated that they were satisfied with the care delivered by PHCWs and that their needs were being addressed at the household level. She said: "it is very good and I am really happy ... because the midwife had used the knowledge that she'd got from the training to my baby and myself" [mother, with daughter age 2 weeks].

Mothers stated they were satisfied because they believed they were getting more attention from the PHCWs. One mother reported:

It is better now ... in the past ... when we went home from hospital after a birth, nothing happened ... no one gave attention (to me and my baby) except my neighbour. Now I am visited by the midwife and CHW ... I am so happy .... [Mother, with son age 4 weeks]]

Another factor found to contribute to mothers' satisfaction was that the interventions delivered by PHCWs had led to improved child health. Three mothers with low birth weight babies reported that their babies' weight had increased significantly within a few weeks after applying the Kangaroo Mother Care technique that had been suggested by the PHCW.

\section{The socio-cultural context}

Despite the successful outcome reported by many mothers, some participants noted issues and preferences in health service delivery that was not consistent with CCM. Some mothers said that they could not accept medication offered by PHCWs for cultural and religious reasons. This was affirmed by a mother who said "I don't want my baby to be injected. My other children have never got immunised ... I believe that the substance of the drug is not halal". ${ }^{l}$.Some mothers reported their preference for using traditional remedies such as herbs for their family members. During a consultation a mother described putting a herbal substance akin to pepper on her baby's umbilical cord. In order to improve health service at village level, mothers expected there would be a nurse or midwife they knew who resided in their village. Two mothers said that if a skilled health worker is available in their village, access to health care could be much improved.

\footnotetext{
${ }^{1}$ A term designated to any object or action, which is permissible to use or engage in, according to Islamic law.
} 


\section{Discussion}

Despite some mothers who found the services provided by PHCWs to be inconvenient, most participants affirmed that the implementation of the CCM program had resulted in better access to care. Mothers reported that they were satisfied with the care provided and they had improved their understanding of danger signs and health practices. These improvements were reported to have contributed to an increase in their compliance to care.

The ultimate goal of implementation of CCM is to reduce infant mortality in the district where the model is employed [5]. Further, the Ministry of Health has provided desired outcomes which include : improved access to interventions offered through CCM; an increase in the care seeking behaviour of community members; and an increase in the quality of child health services [5]. Findings from this research indicate that the intermediate results of CCM implementation have been achieved.

\section{Improved access to care}

The fact that CCM improves the community's access to care in rural areas is supported by earlier studies [11, 21-25]. Although there is a debate concerning whether CCM is the most effective model to reduce infant mortality, all studies agree that the model has enabled the community to gain better access to care through the deployment of PHCWs in rural areas. All studies recommend that for a successful CCM roll out, supportive policy at national and provincial levels should be put in place.

One of the challenges associated with maternal and infant death in rural areas is accessing emergency transport that often lead to delays in seeking and receiving care [26], and the shortage of trained health workers [27]. The CCM model has helped to circumvent many of these delays by the provision of a PHCW at the village level along with the improvement of their capacity in addressing child health problem. The presence of PHCWs in villages can ensure the provision of initial treatment to sick children so that they may not need to travel to health facilities to get timely help. However, such health workers are not always available and in our study mothers indicated that this was a reason why traditional healers were called upon for help.

\section{Improvements in health literacy}

Since the CCM approach was introduced in villages in Kutai Timur, mothers stated that their understanding of illness had improved and they could more readily identify if there was something wrong with their babies and therefore seek help. Participants also agreed that the frequent visit from PHCWs to the home had enhanced the relationship between mothers and PHCWs. This familiarity with the Service resulted in mothers' confidence in accessing support or help in a timely manner from the PHCWs. Mothers also confirmed that they received important lessons from PHCWs so that they could identify health problems when they occur in the future with their own or relatives' babies.

The results of this study concur with the findings from the research conducted by Akweongo et al. [28] that investigated CCM implementation for malaria in children in five sites in Africa. The authors report that caregivers' knowledge about the disease signs and symptoms improved following implementation of nominate the intervention. The improvement of mothers' knowledge of the signs of childhood illness is also demonstrated by George et al. [29] following CCM implementation in Nicaragua. Beyond the studies on improvements in knowledge of CCM, other literature has established an association between child health and parent health literacy and important health outcomes. Two systematic reviews found that children whose parents had low health literacy often displayed poor preventative health care behaviours as adults that lead to poor child health outcomes $[30,31]$. This highlights the need to integrate health promotion strategies into all child health programs that pay particular attention to the literacy needs of families [32], including appropriate resources for families [33]. In addition, literacy programs for poor women may need to take health literacy into consideration [34]. Other interventions such as women's groups may strengthen the CCM model to provide additional opportunities for education and help to encourage families to seek immediate help when an infant is sick [35]. 


\section{Care compliance: Reciprocal benefits}

One finding from this study was that mothers' compliance with care protocols improved following CCM implementation. A study by Winch et al. [36] in Africa and South East Asia, highlights the important role that PHCWs play in monitoring the compliance of the family with regard to the management of pneumonia and malaria. The study revealed that PHCW supervision generated improved compliance with care and treatment. However, not all studies have demonstrated an increase in compliance following the implementation of CCM. Poor compliance with treatment was reported following CCM implementation for childhood malaria in Sierra Leone [37] and Congo [38]. This highlights the need to review community intervention programs that involve CHWs.

Despite the important role of the PHCW in ensuring compliance, family members need to gain appropriate skills, knowledge and behaviours to continue to administer medications as per the required protocol. Therefore, ensuring that sick children receive appropriate therapy at home without constant supervision by PHCWs is essential and can have a positive impact on health workers themselves. Kane et al. [39] reported that the compliance of a mother with prescribed health care was a factor that increased health worker motivation. Mothers' compliance with care may contribute to not only improved child health but can help to make PHCWs feel valued which may have an effect on health worker performance. Improvements in the performance of PHCWs can in turn increase mother's satisfaction with and acceptability of the care they receive.

These reciprocal benefits, that is mothers' increased compliance with care and PHCWs' increased motivation as a result of the relationships, can be explained by 'positive reinforcement' theory [40]. Positive reinforcement involves the addition of a reinforcing stimulus following a behaviour that makes it more likely that the behaviour will occur again [41]. In other words, positive reinforcement relates to any stimulus given to certain actions or behaviours that can increase the motivation of an individual to maintain the desired behaviour [42]. In the context of PHCWs-mother's relationships, the visits made by PHCWs to families had stimulated the mothers to comply with the planned care. Likewise, the compliance of mothers with the care would increase PHCWs' motivation in undertaking their tasks in the community hence feeding the positive feedback loop.

It is argued that ensuring socio-cultural appropriateness is crucial in the introduction of a new model of care. Program implementers should consider the community values and beliefs that may positively influence child health care delivery while at the same time seek to reduce practices that may be harmful. One way forward may be to increase the involvement of traditional healers and birth attendants in the delivery of care. PHCWs may need to better coordinate the delivery of care and information with traditional providers to prevent unsafe practices such as the bathing of newborns within 6 hours after birth or the application of substances to the umbilical stump. This could help to ensure that essential newborn care practices as recommended by WHO are followed to reduce the chances of hypothermia and infection [43].

It is important for program implementers to consider and plan for support for families in incremental changes towards acceptance of the new ideas so that CCM can be culturally acceptable. Furthermore the program material could also be revised in an incremental way to include more culturally appropriate and consonant material over time. Strategies to ensure cultural appropriateness in the adaptation of a new model of care should include: improving the cultural competencies of PHCWs; promoting effective communication between PHCWs and families; strengthening coordination with traditional health healers; and involving families in care plan and care decision [44].

\section{Conclusion}

This paper has introduced the challenges associated with high infant mortality and the problem of inequitable access to healthcare that remains a concern for governments and communities in LMIC. In order to accelerate action towards the reduction of infant mortality in Indonesia, CCM was introduced in the Kutai Timur district. From the context outlined in the paper, it can be seen that the introduction of the new model may provide benefit for the community in rural areas in Indonesia in terms of increasing access to health care, in particular access to lifesaving interventions for sick children. The employment of PHCWs at village level in rural areas appears to have increased mothers' health literacy, their compliance with care protocols and health seeking behaviour. 
More research is needed to identify the long term benefits of the CCM approach and furthermore to adequately resource the model with consideration to the increasing community needs and integrate CCM with other maternal and child health services in Indonesia.

\section{Acknowledgement}

We would like to acknowledge the support of The Directorate General of Higher Education, Ministry of Education and Culture, The Republic of Indonesia who provided a scholarship for the first author to undertake a doctoral program at UTS that investigated CCM in Indonesia. We would also like to thank the staff at the Maternal and Child Health Integrated Program (MCHIP) and the Kutai Timur district health office and the families receiving care who enabled this research to be undertaken. 


\section{References}

1. WHO. Media center: The top 10 causes of death 201322 September 2013]; Available from: http://www.who.int/mediacentre/factsheets/fs310/en/index2.html.

2. The World Bank. Mortality rate, infant (per 1,000 live births). 20169 June 2016]; Available from: http://data.worldbank.org/indicator/SP.DYN.IMRT.IN.

3. Sutan, R. and S. Berkat, Does cultural practice affects neonatal survival-a case control study among low birth weight babies in Aceh Province, Indonesia. BMC pregnancy and childbirth, 2014. 14(1): p. 1.

CORE Group. Who We Are. 201010 October 2010]; Available from: http://coregroup.org/about-us/who-we-are-and-what-wedo.

5. CORE Group, et al., Community Case Management Essentials: Treating Common Childhood illnesses in the Community. 2010, Washington, D.C.

6. Wardlaw, T.M., E.W. Johansson, and M. Hodge, Pneumonia: The forgotten killer of children. 2006, UNICEF/ WHO: New York

7. Kutai Timur District Health Office and MCHIP, District Team Problem Solving to reduce infant mortality and improve maternal health. 2011, Kutai Timur District health Office: Sangatta.

8. Theodoratou, E., et al., The effect of case management on childhood pneumonia mortality in developing countries. International Journal of Epidemiology, 2010. 39: p. 155-171.

9. Wharton-Smith, A., H. Counihan, and C. Strachan, Implementing integrated community case management: stakeholders experieces and lessons learned in three African Countries. www.malariaconcortium/org/learningpapers, 2014.

10. Sazawal, R. and L. Black, Effect of pneumonia case management on mortality in neonates, infants, and preschool children: A meta-analysis of community-based trials. The Lancet Infectious Diseases, 2003. 3(9): p. 547-556.

11. Soofi, S., et al., Effectiveness of community case management of severe pneumonia with oral amoxicillin in children aged 2-59 months in Matiari district, rural Pakistan: a cluster-randomised controlled trial. Lancet, 2012. 379 p. 729-737.

12. Marsh, D.R., et al., Introduction to a special supplement: evidence for the implementation, effects, and impact of the integrated Community Case Management strategy to treat childhood infection. American Journal of Tropical Medicine and Hygiene, 2012. 87(5): p. $2-5$

13. Kutai Timur Government, Sekilas Kabupaten Kutai Timur. 2010.

14. Kutai Timur District Health Office, Kutai Timur Health Profile. 2010, Kutai Timur District Health Office: Sangatta.

15. Simkes UGM, Gambaran sistem informasi dinas kesehatan Kutai Timur provinsi Kalimantan Timur. 2007.

16. Guest, G., A. Bunce, and L. Johnson, How Many Interviews Are Enough?: An Experiment with Data Saturation and Variability. Field Methods, 2006. 18(59).

17. Temple, B. and A. Young, Qualitative research and translation dilemma. Qualitative Research, 2004. 4(2): p. 161-178.

18. Morse, J.M., Emerging from the data: The cognitive processes of analysis in qualitative inquiry, in Critical issues in qualitative research methods, J.M. Morse, Editor. 1994, Sage: Thousand Oaks.

19. Sandelowski, M., Qualitative analysis: What it is and how to begin. Research in Nursing \& Health, 1995. 18: p. 371-375.

20. Peshkin, A., The Nature of Interpretation in Qualitative Research. Educational Researcher, 2000. 29(9): p. 5-9.

21. Ghimire, M., Y.V. Pradhan, and M.K. Maskey, Community-based interventions for diarrhoeal diseases and acute respiratory infections in Nepal. Bulletin of the World Health Organization, 2010. 88: p. 216-221.

22. Nanyonjo, A., et al., Community Acceptability and Adoption of Integrated Community Case Management in Uganda. American Journal of Tropical Medicine and Hygiene, 2012. 87(5): p. 97-104.

23. George, A., et al., Community case management in Nicaragua: lessons in fostering adoption and expanding implementation. Health Policy and Planning 2011. 26: p. 327-337.

24. Sadler, K., et al., Community Case Management of Severe Acute Malnutrition in Southern Bangladesh. 2011, Save the Children, Feinstein International Center: Boston.

25. Young, M., et al., World Health Organization/United Nations Children's Fund Joint Statement on Integrated Community Case Management: An Equity-Focused Strategy to Improve Access to Essential Treatment Services for Children. The American Society of Tropical Medicine and Hygiene, 2012. 87(5): p. 6-10. 

1110 .

27. Titaley, C.R., et al., Challenges to the implementation of the integrated management of childhood illness (IMCI) at community health centres in West Java province, Indonesia. WHO, 2014. 161.

28. Akweongo, P., et al., Feasibility and acceptability of ACT for the community case management of malaria in urban settings in five African sites. Malaria Journal, 2011. 10(1): p. 240.

29. George, A., et al., Community case management of childhood illness in Nicaragua: transforming health systems in underserved rural areas. Journal of health care for the poor and underserved, 2009. 20(4A): p. 99-115.

30. DeWalt, D.A. and A. Hink, Health Literacy and Child Health Outcomes: A Systematic Review of the Literature. Pediatrics, 2009 124(Supplement 3): p. S265-S274.

31. Sanders, L.M., et al., Literacy and child health: A systematic review. Archives of Pediatrics \& Adolescent Medicine, 2009. 163(2): p. 131-140.

32. Sanders, L.M., et al., Health Literacy and Child Health Promotion: Implications for Research, Clinical Care, and Public Policy. Pediatrics, 2009. 124(Supplement 3): p. S306-S314.

33. Hewer, L.-a. and D. Whyatt, Improving the implementation of an early literacy program by child health nurses through addressing local training and cultural needs. Contemporary Nurse, 2006. 23(1): p. 111-119.

34. Nordtveit, B.H., Poverty alleviation and integrated service delivery: Literacy, early child development and health. International Journal of Educational Development, 2008. 28(4): p. 405-418.

35. Lewycka S, et al., Effect of women's groups and volunteer peer counselling on rates of mortality, morbidity, and health behaviours in mothers and children in rural Malawi (MaiMwana): a factorial, cluster-randomised controlled trial. Lancet, 2013. 18(381): p. 1721-1735.

36. Winch, P.J., et al., Intervention models for the management of children with signs of pneumonia or malaria by community health workers. Health Policy and Planning, 2005. 20(4): p. 199-212.

37. Thomson, A., et al., Low referral completion of rapid diagnostic test-negative patients in community-based treatment of malaria in Sierra Leone. Malaria Journal, 2011. 10(94).

38. Hawkes, M., J.P. Katsuva, and C.K. Masumbuko, Use and limitations of malaria rapid diagnostic testing by community health workers in war-torn Democratic Republic of Congo. Malaria Journal, 2009. 8(308).

39. Kane, S.S., et al., A realist synthesis of randomised control trials involving use of community health workers for delivering child health interventions in low and middle income countries. BMC Health Services Research 2010. 10(286).

40. Skinner, B.F., Science and Human Behavior. 1953, New York: Macmillan.

41. Lewis, S.A. and E. Waite. Positive reinforcement. 201215 October 2013]; Available from: http://salewis.iweb.bsu.edu/portfolio/resources/DSN_Project.pdf.

42. Sigler, E.A. and S. Aamidor, From positive reinforcement to positive behaviors: An everyday guide for the practitioner. Early Childhood Education Journal, 2005. 32(4): p. 249-253.

43. WHO, Essential newborn care and breastfeeding. 2003, WHO Regional Office for Europe: Copenhagen.

44. Horton, S. and R.J. Johnson, Improving access to health care for uninsured elderly patients. Public Health Nursing 2010. 27(4): p. $362-370$. 